ARTICLE

https://doi.org/10.1038/s41467-019-08507-4

\title{
Strain engineering in perovskite solar cells and its impacts on carrier dynamics
}

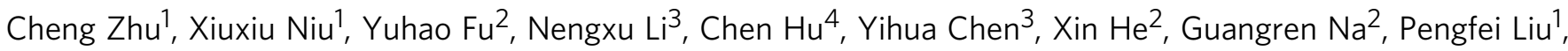 \\ Huachao Zai ${ }^{5}$, Yang Ge ${ }^{6}$, Yue $\mathrm{Lu}^{6}$, Xiaoxing $\mathrm{Ke}^{6}$, Yang Bai ${ }^{1}$, Shihe Yang ${ }^{4}$, , Pengwan Chen ${ }^{8}$, Yujing $\mathrm{Li}^{1}$, \\ Manling Sui id ${ }^{6}$, Lijun Zhang (iD ${ }^{2}$, Huanping Zhou ${ }^{3} \&$ Qi Chen (iD ${ }^{1}$
}

The mixed halide perovskites have emerged as outstanding light absorbers for efficient solar cells. Unfortunately, it reveals inhomogeneity in these polycrystalline films due to composition separation, which leads to local lattice mismatches and emergent residual strains consequently. Thus far, the understanding of these residual strains and their effects on photovoltaic device performance is absent. Herein we study the evolution of residual strain over the films by depth-dependent grazing incident $X$-ray diffraction measurements. We identify the gradient distribution of in-plane strain component perpendicular to the substrate. Moreover, we reveal its impacts on the carrier dynamics over corresponding solar cells, which is stemmed from the strain induced energy bands bending of the perovskite absorber as indicated by first-principles calculations. Eventually, we modulate the status of residual strains in a controllable manner, which leads to enhanced PCEs up to $20.7 \%$ (certified) in devices via rational strain engineering.

\footnotetext{
${ }^{1}$ Beijing Key Laboratory of Construction Tailorable Advanced Functional Materials and Green Applications, Advanced Materials Experimental Center, School of Materials Science \& Engineering, Beijing Institute of Technology, 100081 Beijing, China. ${ }^{2}$ State Key Laboratory of Superhard Materials, Key Laboratory of Automobile Materials of MOE, and School of Materials Science and Engineering, Jilin University, 130012 Changchun, China. ${ }^{3}$ Department of Materials Science and Engineering, College of Engineering, Peking University, 100871 Beijing, China. ${ }^{4}$ Department of Chemistry, The Hong Kong University of Science and Technology, Clear Water Bay, Kowloon, Hong Kong, China. ${ }^{5}$ Department of Materials Science and Engineering, College of Science, China University of Petroleum, 102249 Beijing, China. ${ }^{6}$ Institute of Microstructure and Properties of Advanced Materials, Beijing University of Technology, 100124 Beijing, China. ${ }^{7}$ Guangdong Key Lab of Nano-Micro Material Research, School of Chemical Biology and Biotechnology, Shenzhen Graduate School, Peking University, Xili University Town, 518055 Shenzhen, Guangdong, China. ${ }^{8}$ State Key Laboratory of Explosion Science and Technology, Beijing Institute of Technology, 100081 Beijing, China. These authors contributed equally: Cheng Zhu, Xiuxiu Niu, Yuhao Fu. Correspondence and requests for materials should be addressed to L.Z. (email: lijun_zhang@jlu.edu.cn) or to H.Z. (email: happy_zhou@pku.edu.cn) or to Q.C. (email: qic@bit.edu.cn)
} 
O ver the past decade, hybrid organic-inorganic halide perovskites have received enormous interest as low-cost and highly efficient light absorbers in photovoltaics ${ }^{1-6}$. The power conversion efficiency (PCE) of perovskite solar cells has shortly surpassed $22 \%$ in the lab scale ${ }^{7}$, and that of larger-area $\left(>1 \mathrm{~cm}^{2}\right)$ devices has exceeded $20 \%^{8-11}$. The highest PCEs are mostly achieved by employing the mixed halide perovskites (e.g., $\left(\mathrm{HC}\left(\mathrm{NH}_{2}\right)_{2} \mathrm{PbI}_{3}\right)_{0.85}\left(\mathrm{CH}_{3} \mathrm{NH}_{3} \mathrm{PbBr}_{3}\right)_{0.15}$ i.e., $\left(\mathrm{FAPbI}_{3}\right)_{0.85}$ $\left.\left(\mathrm{MAPbBr}_{3}\right)_{0.15}\right)$. Through element substitution, it provides a largely unexplored compositional space to tailor the physiochemical properties of corresponding materials for efficient and stable devices ${ }^{1,12-15}$. However, the mixed hybrid perovskites potentially suffer from materials inhomogeneity partially due to composition separation ${ }^{16,17}$ and/or thermal stress. This may be originated from substantial chemical mismatch among each component, and the nonequilibrium growth conditions during film fabrication. On one hand, serious material inhomogeneity is regarded as phase separation, deviating from the originally desired materials properties to deteriorate the resultant device performance (both efficiency and operational stability) ${ }^{18,19}$. On the other hand, moderate material inhomogeneity correlates to local lattice mismatches and emergent residual strains in perovskite films. It deserves more attention, because such residual strains should result in lattice distortion of microscopic crystal structure, and further affect optoelectronic properties of the perovskite thin film ${ }^{20-25}$.

Recently, the residual strains have been identified in the singlecomposition $\mathrm{MAPbI}_{3}$ polycrystalline films, and were found to influence stability of perovskite films under illumination ${ }^{22}$. In an even wider spectrum of semiconductors, strain has been extensively investigated, and accordingly, strain engineering has been exploited to tailor the optoelectronic functionalities. For instance, the application of tensile strain was found to improve light emission in Germanium crystals and drive indirect-to-direct optical transition in bilayered two-dimensional $\mathrm{WSe}_{2}{ }^{26-28}$. Strain compensation strategy has been demonstrated to improve efficiencies of GaAs-based quantum dot solar cells ${ }^{29,30}$. Strainengineered $\mathrm{MoS}_{2}$ monolayer was also proposed to have broadrange capture of solar energy ${ }^{26}$. For hybrid halide perovskites, especially mixed perovskites, in-depth understanding of their characteristics and effects on the material's optoelectronic properties, is still absent. This precludes effective employment of strain engineering to further enhance the device performance.

In this report, we probe the residual strain distribution profiles in the mixed perovskite thin films and its effects on photovoltaic device efficiency. We investigate the evolution of in-plane residual strain over the film thickness in the typical mixed perovskite $\left(\mathrm{FAPbI}_{3}\right)_{0.85}\left(\mathrm{MAPbBr}_{3}\right)_{0.15}$ by using grazing incident X-ray diffraction (GIXRD) measurement. We identify a gradient distribution of in-plane strain component that correlates to the composition inhomogeneity perpendicular to the substrate. We further demonstrate a feasible method to modulate the tensile or compressive nature of residual strain and even its gradient over perovskite films in a controllable manner. With the aid of firstprinciples calculations, we find that the strain gradient induces energy bands bending and thus affects the carrier dynamics across the interfaces over the solar cell. By deliberately engineering the residual strains to enhance carrier extraction efficiency, we successfully fabricate strain-engineered perovskite solar cells to achieve enhanced PCEs up to $20.7 \%$ (certified).

\section{Results}

Probing residual strain gradient of mixed perovskite films. In the direction parallel to substrates, the grain-to-grain inhomogeneity in polycrystalline films was already observed by adopting photoluminescence in a scanning electron microscopy (PL-SEM) and conductive-atomic force microscopy (C-AFM) ${ }^{31}$. However, it lacks depth profile along the film thickness regarding the lattice structure inhomogeneity. At the macroscopic level, the vertical homogeneity of thin films can be quantitatively evaluated by residual strain to reflect the lattice mismatch ${ }^{20,22,32}$. The macroscopic residual strain is an internal strain in polycrystalline materials that is balanced over a wide range of grains. We explored the residual strain distribution for mixed perovskite films vertically with the depth-dependent GIXRD measurement, wherein the classical $\sin ^{2} \varphi$ measurement is combined with grazing incident $\mathrm{X}$-ray diffraction to probe the in-plane residual strain. This method has been used in thin films of $\mathrm{ZrO}_{2}$ and TiN, which provides reliable depth resolution to reveal lattice structure evolution ${ }^{33-35}$. As depicted in Fig. 1a, we fixed the $2 \theta$ and varied the instrument tilt angle $\psi$ to obtain corresponding X-ray diffraction (XRD) patterns as shown in Fig. 1b. For detailed test information, please see the Supplementary method, Supplementary Fig. 1 and Supplementary Table 1. The mixed perovskite films on the $\mathrm{SnO}_{2} / \mathrm{ITO} /$ glass substrates showed XRD peaks $(2 \theta)$ at around $14.0^{\circ}, 31.6^{\circ}$, and $40.5^{\circ}$, corresponding to (001), (012), and (022) crystallographic planes, respectively ${ }^{36}$. Among all three planes with intensive diffraction peaks, the (012) plane was chosen for further analysis due to its high diffraction angle and multiplicative factor, which provides the most reliable structure symmetry information.

We picked up three representative depths of 50, 200 and 500 $\mathrm{nm}$ to check the residual strain in the mixed perovskite films with the nominal formula of $\left(\mathrm{FAPbI}_{3}\right)_{0.85}\left(\mathrm{MAPbBr}_{3}\right)_{0.15}$. At each fixed depth, diffraction data were fitted with Gaussian distribution function and it clearly observed a systematic shift in peak position to lower $2 \theta$ when the penetrated depth increased (Fig. $1 \mathrm{~b}$ and Supplementary Fig. 2). Generally, $\sin ^{2} \varphi$ and $2 \theta$ follow a linear relationship and the slope of the fitting line stands for the magnitude of the residual strain (see equation (1) in Supplementary method). As shown in Fig. 1b, all fitting lines exhibited negative value in the slopes, which means (012) crystal planes exhibit enlarged distance when turning to the out-of-plane direction at the same detected depth. It thus indicates the entire sample is subjected to tensile strain. It is in agreement with previous study wherein the residual tensile strain was observed in perovskite thin films upon the similar annealing process ${ }^{22}$.

With the increase of probe depth, the fitting lines show smaller slopes in absolute value, which implies the macroscopic residual tensile strain gradually decreases. Moreover, we found the most significant deviation in lattice constant at $50 \mathrm{~nm}$ as compared to that of 200 and $500 \mathrm{~nm}$. Besides, the (001) and (022) crystal planes were also found to follow the similar trend (Supplementary Fig. 2). It indicates the residual strain inhomogeneity in the mixed perovskite thin film, wherein the top surface of the film bears the largest tensile strain. It is worth noting that residual strain correlates to the lattice distortion, which might affect the carrier dynamics at the relevant interfaces, as will be discussed later. Therefore, the residual tensile strain gradient was clearly identified, which gradually decreases from the top surface to the core in the mixed perovskite polycrystalline thin films.

So far, we have observed the gradient distribution of tensile strain in the perovskite thin film. And it will be interesting to understand the origin of the residual strain. The residual stain is often stemmed from the lattice mismatch due to lattice structure evolution. We thus conducted GIXRD measurements with variable grazing incident angle $\omega$ (Supplementary Table 1) to reveal the lattice mismatch along the film thickness. These depthdependent XRD patterns were roughly similar and no new diffraction peaks appeared, indicating the film exhibited the same cubic phase structure at different depths. However, we observed a 
a
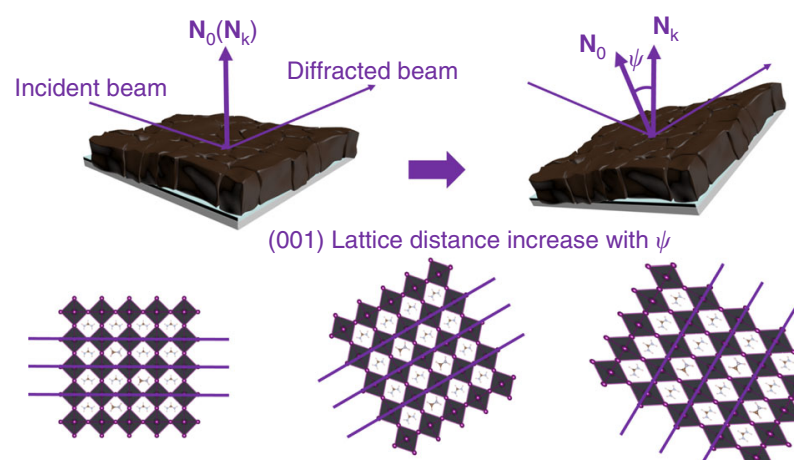

(001) Lattice distance increase with $\psi$
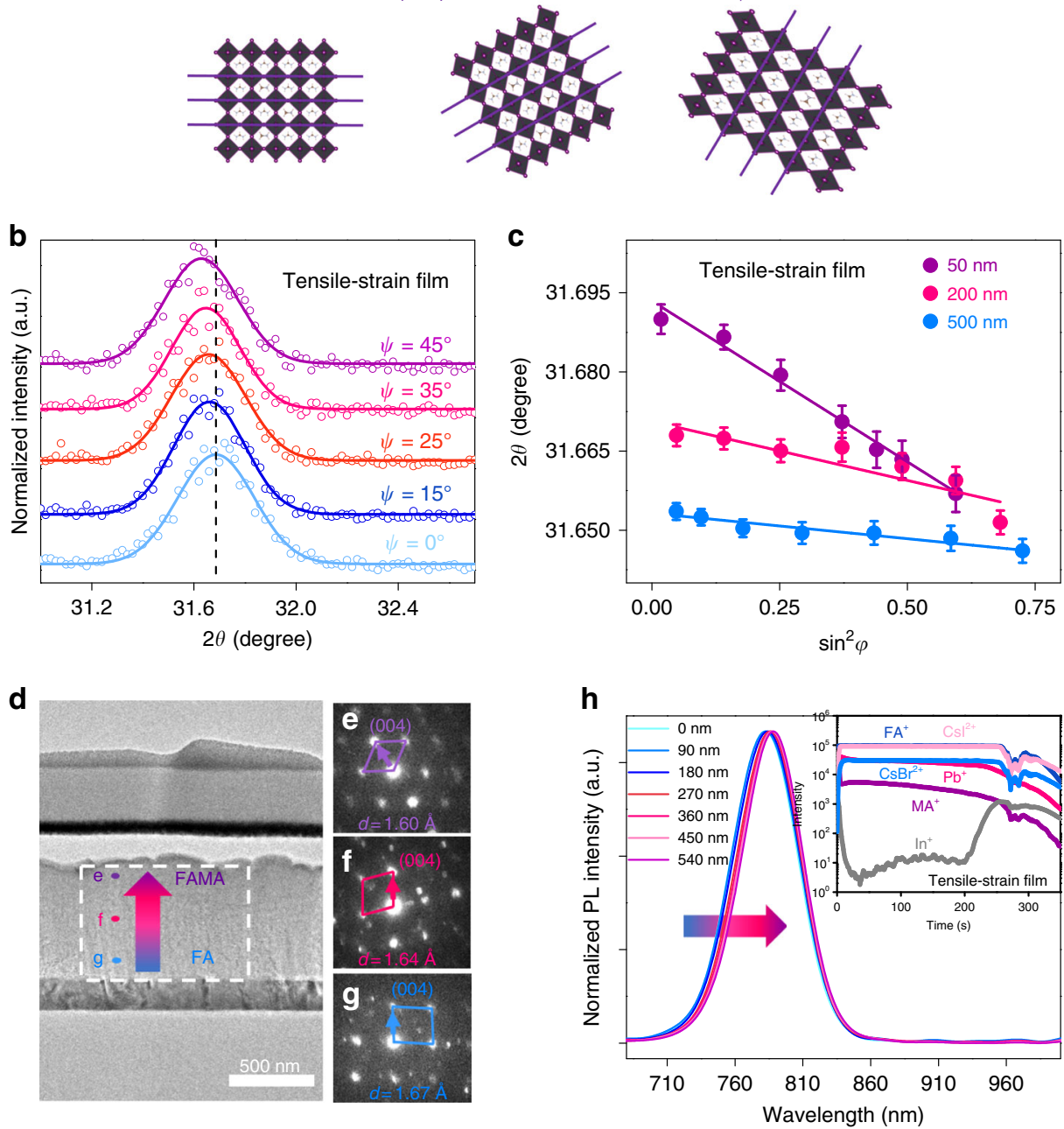

Fig. 1 Gradient lattice structure characterization. a Schematic illustration of the residual strain distribution measurement. The corresponding XRD patterns and lattice structure strain information can be obtained by fixing the test crystal plane and adjusting the instrument tilt angle $\psi$, where $\mathbf{N}_{0}$ is the sample normal direction and $\mathbf{N}_{\mathrm{k}}$ is the diffraction vector. $\mathbf{b}$ GIXRD spectrum at different tilt angles at the depth of $50 \mathrm{~nm}$ for the tensile-strained film. c Residual strain distribution in the depth of 50,200,500 nm for the tensile-strained film (measured (points) and Gauss fitted (line) diffraction strain data as a function of $\sin ^{2} \varphi$ ). The error bar indicates standard deviation of the $2 \theta$. $\mathbf{d}$ The cross-sectional TEM image of device. e, $\mathbf{f}, \mathbf{g}$ The nano-beam electron diffraction patterns ([100] zone axis and TEM specimens is FIBed), corresponding with e-f-g point in d, confirming the FAMA hybrid perovskite phase structure transform to nearly pure FA phase from the surface to the bottom of perovskite film according to the larger quadrangle. $\mathbf{h}$ PL depth profile of confocal fluorescence microscope, the inset represents TOF-SIMS depth profiles of the $\left(\mathrm{FAPbl}_{3}\right)_{0.85}\left(\mathrm{MAPbBr}_{3}\right)_{0.15}$ perovskite film with tensile strain. XRD $\mathrm{X}$-ray diffraction, TEM transmission electron microscopy

systematic shift of diffraction peaks along with the probing depths at 50,200 , and $500 \mathrm{~nm}$ in the perovskite film. Take (001) planes for example, the corresponding diffraction peaks for 50,200 , and $500 \mathrm{~nm}$ exhibited peak centers at $14.01^{\circ}, 13.94^{\circ}$, and $13.90^{\circ}$, respectively (Supplementary Fig. 3). Among different crystal planes of (001), (012) and (022), corresponding peak positions gradually shift towards the low diffraction angle with the increase of probing depths. According to the Bragg's law, larger lattice constant was expected in the deeper zone of films. Thus, the crystal structure inhomogeneity along the film thickness is detected unambiguously. To provide the evidence regarding the structure inhomogeneity in the microscopic level, transmission electron microscopy (TEM) nano-beam electron diffraction measurement was further carried out to investigate crystal structure evolution along the depth direction of mixed perovskite films. We obtained the high-resolution TEM image with microarea diffraction patterns to inspect three typical regions with different depths (Fig. 1d-f). After careful calibration of all measured diffraction patterns (Supplementary Table 2), we found that the crystal plane distance increased with scanning depth. As shown in Fig. 1e-g, lattice parameters of (004) planes were measured to be $1.60,1.64$ and $1.67 \AA$ for three individual areas, respectively, which is consistent with the GIXRD results. Combining the microscopic analysis (TEM) and the long-range structure characterization (GIXRD), we clearly illustrate lattice structure evolution developed vertically in the mixed perovskite 
polycrystalline thin films, wherein the lattice constant decreases from the surface to the bottom.

The above-mentioned crystal structure inhomogeneity is likely to correlate to the composition evolution in mixed perovskite thin films. It is commonly accepted that $\mathrm{FAPbI}_{3}$ exhibits the cubic phase with the lattice constant of $6.357 \AA^{36}$. When MA and/or Br element are incorporated to form the mixed perovskites single crystals, they hold the cubic phase with decreased lattice parameters. We observed smaller lattice constant at the surface of the film, which may partially attribute to higher ratio of MA and/or Br components in the long-range ordered FA based perovskite lattices. To identify the distribution of MA and/or $\mathrm{Br}$ components, we resorted to time-of-flight secondary ion mass spectrometry (TOF-SIMS) depth profiles and TEM/EDX mapping for the $\left(\mathrm{FAPbI}_{3}\right)_{0.85}\left(\mathrm{MAPbBr}_{3}\right)_{0.15}$ perovskite film samples as shown in the inset of Fig. 1h, Supplementary Figs. 4 and 5. It shows a homogeneous distribution of $\mathrm{CsBr}^{2+}, \mathrm{CsI}^{2+}, \mathrm{FA}^{+}$in the all samples, which indicates the even distribution of the halogen $\mathrm{Br}^{-}$. It is reasonable because the smallest halogen ion $\mathrm{Br}^{-}$can diffuse and be evenly distributed during the crystallization process. To be noted, the signal intensity of $\mathrm{MA}^{+}$fragment decreased substantially from the surface to the core region in all mixed perovskite thin films, consistent with the XRD and TEM results. Unambiguously it reveals the gradient evolution of composition and thus lattice structure across the depth direction due to the gradient distribution of organic cation $\mathrm{MA}^{+}$. The nonuniform composition distribution indicates the unique kinetics for film growth, which is possibly related to the coordination strength of different precursors ${ }^{37}$ and film processing conditions. It clearly implies the compositional distribution serve as one major factor that leads to the gradient residual strain.

It is revealed that hybrid perovskites with different lattice structures often exhibit different optoelectronic properties ${ }^{25,38}$ and it is expected to observe the gradient variation in terms of optoelectronic properties within the gradient phase structure of the film. Therefore, we examined the depth-dependent photoluminescence (PL) spectra within the film by using confocal fluorescence microscope. With the increasing depth of the beam probe, a systematic red shift of PL spectra was observed (Fig. 1h and Supplementary Fig. 6). Fitting with the Gaussian distribution function, we found the PL peak positions shifted from 781 to 788 $\mathrm{nm}$, and their full width at half maximum (FWHM) decreased along the film thickness. Since the emission photon energy is determined by the bandgap of semiconductors, it indicates the perovskite film exhibits gradually decreased bandgap from the surface to the bottom vertically. To be noted, it follows the similar trend in gradient evolution of lattice structure and compositional distribution, wherein more $\mathrm{MA}^{+}$ions are incorporated in the perovskite crystals at the top surface of the film. In addition, narrower PL linewidth emission at the deeper region of perovskite film was observed, which may indicate weakened interaction between charge carriers and lattice vibrations (phonons) due to improved film homogeneity and lattice order ${ }^{39,40}$.

Modulating the residual strains. Based on the analysis above, we reveal that the observed residual strain gradient in perovskite films is closely related to lattice structure evolution due to detectable compositional inhomogeneity. However, it may not be the only contributor that governs the residual strain, given the largest tensile strain concentrated on the film surface. Interestingly, when examining the pure $\mathrm{MAPbI}_{3}$ perovskite thin film, we still observed the existence of gradient residual strain (Supplementary Fig. 7). It is thus speculated that the thermal strain may take effects due to the temperature gradient during perovskite film fabrication. To illustrate, the perovskite film is roughly divided into two regions, e.g., the surface and the bottom (near the substrate). When heated on a hot plate, both regions experienced substantial temperature gradient (higher at the bottom). Consequently, the inorganic framework near the bottom expands more in volume upon annealing as compared to that at the surface, which facilitates the insertion of larger cation $\left(\mathrm{FA}^{+}\right)$ to form the perovskite crystalline structure. It possibly results in the inhomogeneous composition in the film, wherein $\mathrm{MA}^{+}$prefers to stay at the surface. Upon cooling, it is reasonable that the surface cools down much faster than that of the core, which leads to less volume shrinkage. As a result, the surface withstands tensile strain and the core is subjected to compressive strain accordingly. Additionally, since the thermal expansion coefficient of perovskites is much larger than that of the substrate, the entire film bears the tensile strain during film cooling process. Thereby, the core experiences not only compressive strain from the surface, but also tensile strain from the substrate, which leads to the gradient residual tensile strain in the perovskite thin film.

To verify the speculation, we attempted to modulate the gradient in-plane residual strain in the perovskite thin films by adjusting the annealing process. We first tried to anneal the film at $150^{\circ} \mathrm{C}$ high temperature for a long time, which is a conventional approach to eliminating residual strains in metal alloy materials. Unfortunately, it led to the significant occurrence of $\mathrm{PbI}_{2}$ (Supplementary Fig. 7) in the resultant thin films due to the relative poor thermal stability of hybrid perovskites ${ }^{41}$. We then tried to change the temperature gradient when the films were annealed. Simply, we modified the heat treatment process by flipping over the thin film sample to provide an invert temperature gradient. Interestingly, tensile strain was significantly reduced in the samples upon this treatment as shown in Fig. 2a, b. With small strain gradient and the closer lattice distance in the perovskite film, the lattice structure was almost homogeneous. It thus suggests an effective way to significantly reduce the tensile strain inhomogeneity across the film thickness by tuning the temperature gradient during film processing. Furthermore, we tried to directly perform flipped annealing process to the intermediate state film after the spin-coating process ends, which is expected to apply compressive strain with vertical gradient over the film. In contrast to the previous samples, the as-prepared films show the fitting curves possessed slopes in positive values (Fig. 2c, d), which increased along with the probe depth. It clearly indicates films exhibit compressive strain, which is also distributed in a vertical gradient. Unfortunately, we observed a lot of pinholes in the resultant film possibly because the solvent cannot be spread smoothly during the annealing process (Supplementary Fig. 9). Therefore, we demonstrate the temperature gradient is also an important source to residual strain during the film growth, which further suggests a method to manipulate the evolution of lattice structure of polycrystalline thin films and the surface strain.

To investigate whether the upper contacting layer influences the surface strain, we prepared a tensile-strained film to test the surface residual strain as a reference point. Then for the same sample, $1 \mathrm{~mL}$ chlorobenzene solution was dripped on the film surface during the spin-coating process and the film was tested. Furthermore, the same sample was coated with Spiro-OMeTAD layer and then tested again. We found the same slope of the fitting curves indicating the magnitude of the surface strain remained constant. (Supplementary Fig. 10) It clearly shows that the chlorobenzene dripped process and/or Spiro-OMeTAD contacting layer does not affect the surface strain significantly.

The residual strain and its gradient distribution reflects the structure inhomogeneity in the perovskite thin film along the vertical direction. It is known that the strain gradient is derived from the XRD peak offset, which actually reveals the variation of 
lattice parameters leading to the crystal structure mismatch across the thin film. In hybrid halide perovskites, the framework of corner-sharing $\mathrm{PbI}_{6}$ octahedral contributes to the electronic configuration, especially at the band-edge ${ }^{42,43}$. It means variations in the inorganic framework would possibly result in the change in optoelectronic properties of the materials. The structure variations include enlargement/shrinkage, tilting, and other deformation of the octahedral network, which can be clearly illustrated by measuring the residual strain. Therefore, it is of great interest to bridge the gulf between the residual strain and the optoelectronic properties of the materials and relevant devices. Given the thin film surface exhibits the most significant strain, it is reasonable focus on the carrier dynamic behavior across the interface via strain modulation, and their effects on device performance.

Impacts of strain on carrier dynamics and device performance. To investigate the impact of the gradient residual strain on the device performance, we first fabricated planar heterojunction solar cells by adopting the perovskite absorbers with/without residual strains. The device architecture follows the regular structure of $\mathrm{ITO} / \mathrm{SnO}_{2} /$ perovskite/Spiro-OMeTAD/Ag. We then compare the $J-V$ curves of the tensile-strained and the strain-free devices. To avoid possible misleading due to sample variation, we fabricated 40 cells under optimal conditions in each batch. Figure $3 \mathrm{a}$ shows the histograms of PCEs for each batch of samples with/without strain. The tensile-strained devices exhibited the PCE averaged around $18.7 \%$ with a wider distribution from $17.3 \%$ and $20.3 \%$. In comparison, the strain-free devices achieved the averaged PCE of $19.8 \%$, whose PCEs were distributed in a narrow range between $18.8 \%$ and $20.7 \%$. The narrow distribution in PCEs of the strain-free devices stands for the good processing reproducibility. We also conducted the current-voltage $(I-V)$ measurement for devices under different annealing conditions to preserve different tensile strains in the absorbers, which provided the statistics of parameters in Supplementary Fig. 11 and Supplementary Table 4 . It is found that the fill factor (FF) and the open-circuit voltage $\left(V_{\mathrm{OC}}\right)$ have significantly improved with the increase of the flipped annealing time, wherein the surface tensile strain is gradually released through prolonged annealing at $120^{\circ} \mathrm{C}$

We measured current-density voltage $(J-V)$ characteristics of one of the best devices under a simulated illumination of air mass (AM) 1.5 and $100 \mathrm{~mW} \mathrm{~cm}^{-2}$. This device generated a short-circuit current density $\left(J_{\mathrm{SC}}\right)$ of $22.8 \mathrm{~mA} \mathrm{~cm}^{-2}$, a $\mathrm{FF}$ of $78.0 \%$, an opencircuit voltage $\left(V_{\mathrm{OC}}\right)$ of $1.17 \mathrm{~V}$, and a power conversion efficiency of $20.7 \%$ (Fig. 3b). The forward and reverse scanning current density-voltage $(J-V)$ curves showed negligible hysteresis in the corresponding device in Supplementary Fig. 11e, which was likely attributed to the improved carrier extraction at the interface ${ }^{44,45}$. By holding a bias near the maximum power output point $(0.96 \mathrm{~V})$, a stabilized photocurrent of $21.3 \mathrm{~mA} \mathrm{~cm}^{-2}$ was obtained, corresponding to a stabilized efficiency of $20.5 \%$ (the inset of Fig. $3 \mathrm{~b}$ ). The device performance was certified by the independent third party (Supporting Information). The External quantum efficiency (EQE) spectra for the two types of devices were shown in Supplementary Fig. 11f. Compared to the tensile strain device, the strain-free device showed improved light harvesting efficiency along the entire absorption wavelength range of 350 to $800 \mathrm{~nm}$. The integrated photocurrent densities were calculated to be $20.81,22.7 \mathrm{~mA} \mathrm{~cm}^{-2}$, respectively, which was in good agreement with the $J_{\mathrm{SC}}$ derived from the $J-V$ measurement. Thus far, we observed the significant improvement in FF and $V_{\mathrm{OC}}$ in strain-engineered devices. This is likely attributed to improved carrier extraction dynamics around the absorber interface, which is subjected to further analysis.
Firstly, the carriers transport behavior at the interface was probed with transient photocurrent (TPC) and time-resolved photoluminescence (TRPL) measurement. The TPC measurement (Fig. 3c) was often used to monitor the carrier transport across the device. By fitting with the exponential function, the photocurrent decay time was significantly reduced from 12.96 to $1.0 \mu$ s (Supplementary Table 5). A faster decay of photocurrent than the reference device suggested the improvement in carrier extraction when tensile strain gradient was almost eliminated at the surface. Further investigation with the TRPL measurement indicates improved hole extraction due to elimination of residual strain, as observed in the samples with the configuration of glass/ perovskite/Spiro-OMeTAD. As shown in Supplementary Fig. 12 and Supplementary Table 6 , the average decay time $\tau_{\text {avg }}$ related to PL quenching was dramatically decreased from 14.6 to $4.9 \mathrm{~ns}$ in the strain-free sample, showing the higher quenching rate. It indicates that the strain-free sample exhibits better hole extraction at the interface between absorber and hole transport material (HTM), as compared to that of the tensile strain sample. This result is in accordance with the TPC measurement, showing that modulation of residual tensile strain can accelerate the carrier transfer process.

Secondly, we investigated how the carrier recombination process of devices is affected by the residual strain. We conducted the combined measurements of light-intensity-dependent $V_{\mathrm{OC}}$, electrochemical impedance spectroscopy (EIS) and transient photovoltage decay (TPV). The light-intensity-dependent $V_{\mathrm{OC}}$ provides critical insights into the mechanism of recombination processes in solar cells ${ }^{46}$. The corresponding charge carrier recombination process is reflected by the ideality factor of " $n$ " as determined by the slope of the $V_{\mathrm{OC}}$ versus incident light-intensity according to the equation $V_{\mathrm{OC}}=E_{\mathrm{g}} / q n k_{\mathrm{B}} T / q \ln J_{0} / J$; where $q$ is the elementary charge, $k_{\mathrm{B}}$ is the Boltzmann constant, and $T$ is the temperature. When the ideality factor $n$ approaches 2, ShockleyRead-Hall (SRH) type, trap-assisted recombination dominates. As shown in Fig. 3d, from the relationship between $V_{\mathrm{OC}} \sim \ln (\mathrm{I})$, the ideality factor $n$ are 1.01, 1.55 for the devices with/without tensile strain, respectively. It indicates that trap-assisted SRH recombination is effectively suppressed by reducing the tensile strain that is mainly located at the absorber surface. The alleviated SRH recombination may be attributed to the reduced trap density in strain-free devices, wherein crystal structure homogeneity is achieved.

The carrier dynamics across the perovskite/HTM interface upon strain modulation is further examined by EIS. Corresponding Nyquist plots were obtained from solar cells with/without gradient residual strain in dark without applied bias (Fig. 3e). It shows two separate arcs, equivalent to the resistive and capacitive components of various interfaces ${ }^{47,48}$. Generally, the first arc in the intermediate-frequency region is associated with the recombination impedance due to the selective contacts or their interface with the perovskite active layer in the device. Here, we mainly focused on the mid-frequency region and found that the recombination impedance increased from 23.69 to $521.10 \mathrm{~K} \Omega$ when the tensile strain was relaxed. It is consistent with that of the light-intensity-dependent $V_{\mathrm{OC}}$ measurement. We further measured the recombination resistance $\left(R_{\text {rec }}\right)$, wherein the device was subjected to applied bias ranging from 0.4 to $1.0 \mathrm{~V}$. By fitting with the simple RC equivalent circuit, we exacted the recombination resistance under different bias as shown in Fig. $3 \mathrm{f}$. It is clear that strain-free devices display a larger $R_{\text {rec }}$ than that of the tensile-strained one, which indicates that the interfacial charge recombination is suppressed in strain-free device. The reduced recombination loss was further confirmed by TPV measurement ${ }^{49}$, wherein the photovoltage decay time was significantly increased from 4.01 to $10.32 \mu \mathrm{s}$ (Supplementary Fig. 12 and 

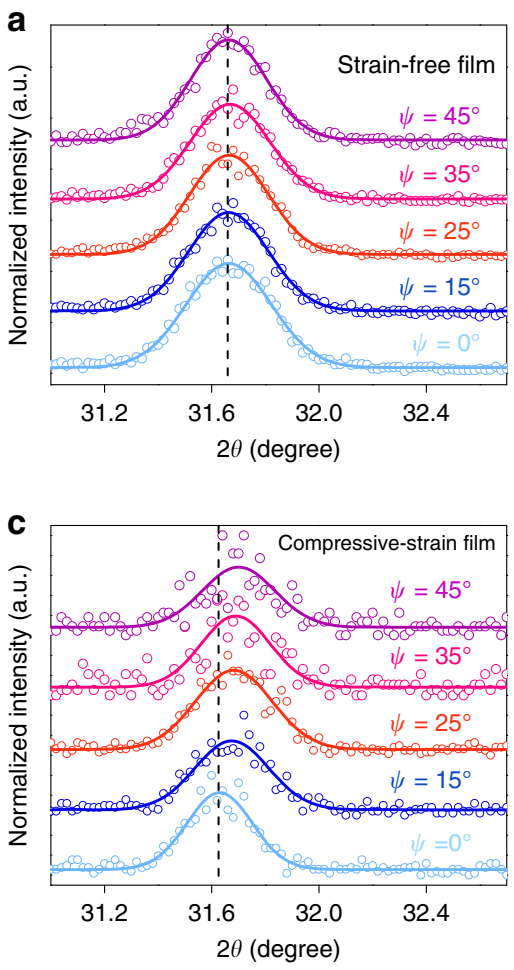
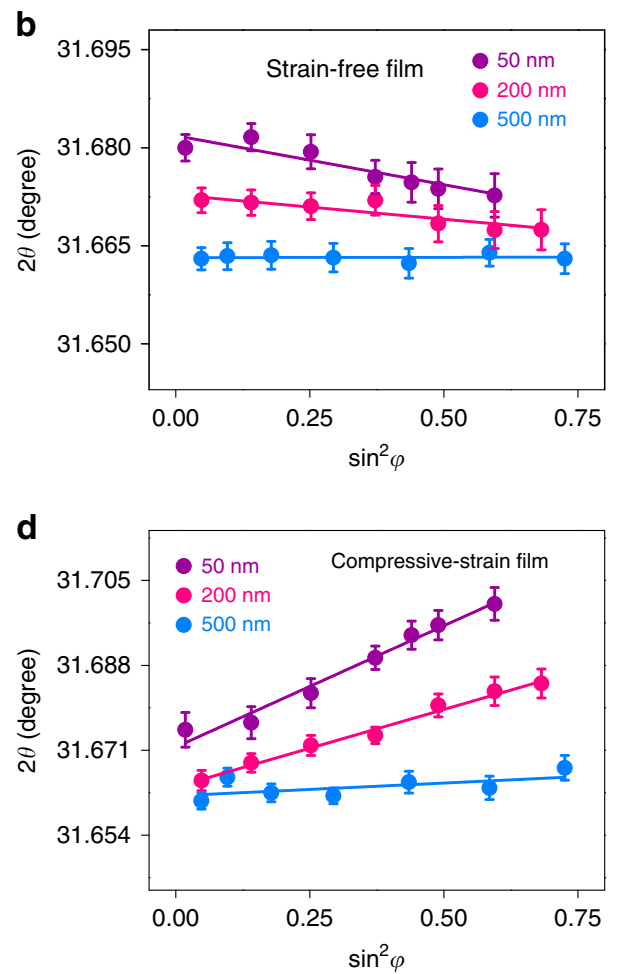

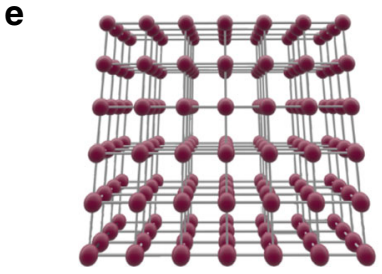

Tensile-strain

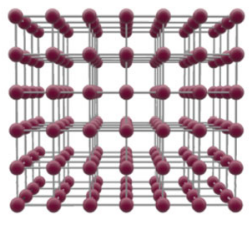

Strain-free

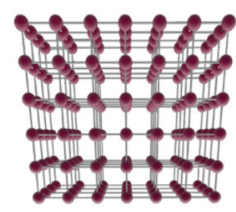

Compressive-strain

Fig. 2 Residual strain distribution measurement with the GIXRD method. a, c GIXRD spectrum at different tilt angles at the depth of $50 \mathrm{~nm}$ for the strainfree film, compressive strained film. b, d Residual strain distribution in the depth of 50, 200, $500 \mathrm{~nm}$ for the strain-free film, compressive strained film (measured (points) and Gauss fitted (line) diffraction strain data as a function of $\sin ^{2} \varphi$ ). The error bar indicates standard deviation of the $2 \theta$. e The schematic representation of the tensile strain state of the film in the top surface, showing the lattice structure with/without tensile strain on the film surface from the perspective of long-range order

Supplementary Table 5) when tensile strain was released. It is also in agreement with higher $V_{\mathrm{OC}}$ observed in the devices with strainfree absorbers.

Mechanisms of the effect of strain on photovoltaic properties. The above results unambiguously indicate that the gradient distribution of residual strains directly affects the hole carrier dynamics across the perovskite/HTM interface and thus the device performance. To reveal the underlying mechanism, we performed first-principles calculations to simulate changes of electronic structure and optoelectronic properties of the perovskite films under strained condition. In particular, we chose the (001)-oriented $\mathrm{FAPbI}_{3}$ perovskite film represented by six-layers Ruddlesden-Popper phase of $\mathrm{FAPbI}_{3}$ and applied in-plane biaxial strains to it. Since (001) is one of predominate growth direction of perovskite crystals as indicated by the XRD measurements, and $\mathrm{FAPbI}_{3}, \mathrm{MAPbBr}_{3}$ and their mixture have similar electronic structures ${ }^{50,51}$, we expect the above model can reasonably mimic the strained mixed perovskite $\left(\mathrm{FAPbI}_{3}\right)_{0.85}\left(\mathrm{MAPbBr}_{3}\right)_{0.15}$ films in experiment. We embedded the $(001) \mathrm{FAPbI}_{3}$ film in the vacuum and applied biaxial strains of $1 \%, 0.5 \%$ (compressive) and $-0.5 \%$,
$-1 \%$ (tensile), respectively (Supplementary Fig. 13). We found that the perpendicular direction exhibit quite small opposite stain components as the rotation of organic FA/MA molecules contributes substantially to strain relaxation. The calculated band structures under tensile, zero, and compressive strains are shown in Fig. 4a, b. One sees that the band gaps of the films show increase with the strain changing from compression, zero-strain, to tension. This agrees with the observed tendency of bandgap change from the ultraviolet (UV)-visible (Vis) absorption and PL measurement (Fig. 4c). To be noted, the bandgap measured from $\mathrm{UV}-\mathrm{V}$ is absorption and PL spectra less than the theoretical value. It is because the theoretical value was calculated with the assumption that the entire lattice structure is subjected to strain state. In our experiment however, the largest strain only locates at the surface region rather than the entire film (Fig. 1c), wherein quite a large portion of films remain the original bandgap. The tendency can be explained by consideration of the band-edge features of $\mathrm{Pb}$ halide perovskites $\mathrm{APbX}_{3}$, where the valence band (VB) edge is composed of the strong anti-bonding interaction between $\mathrm{Pb}-s$ and $\mathrm{X}-\mathrm{p}$ orbitals, and the conduction band (CB) edge is predominantly from $\mathrm{Pb}-\mathrm{p}$ orbital with weak anti-bonding character ${ }^{51,52}$. With the perovskite film experiencing compressive, 

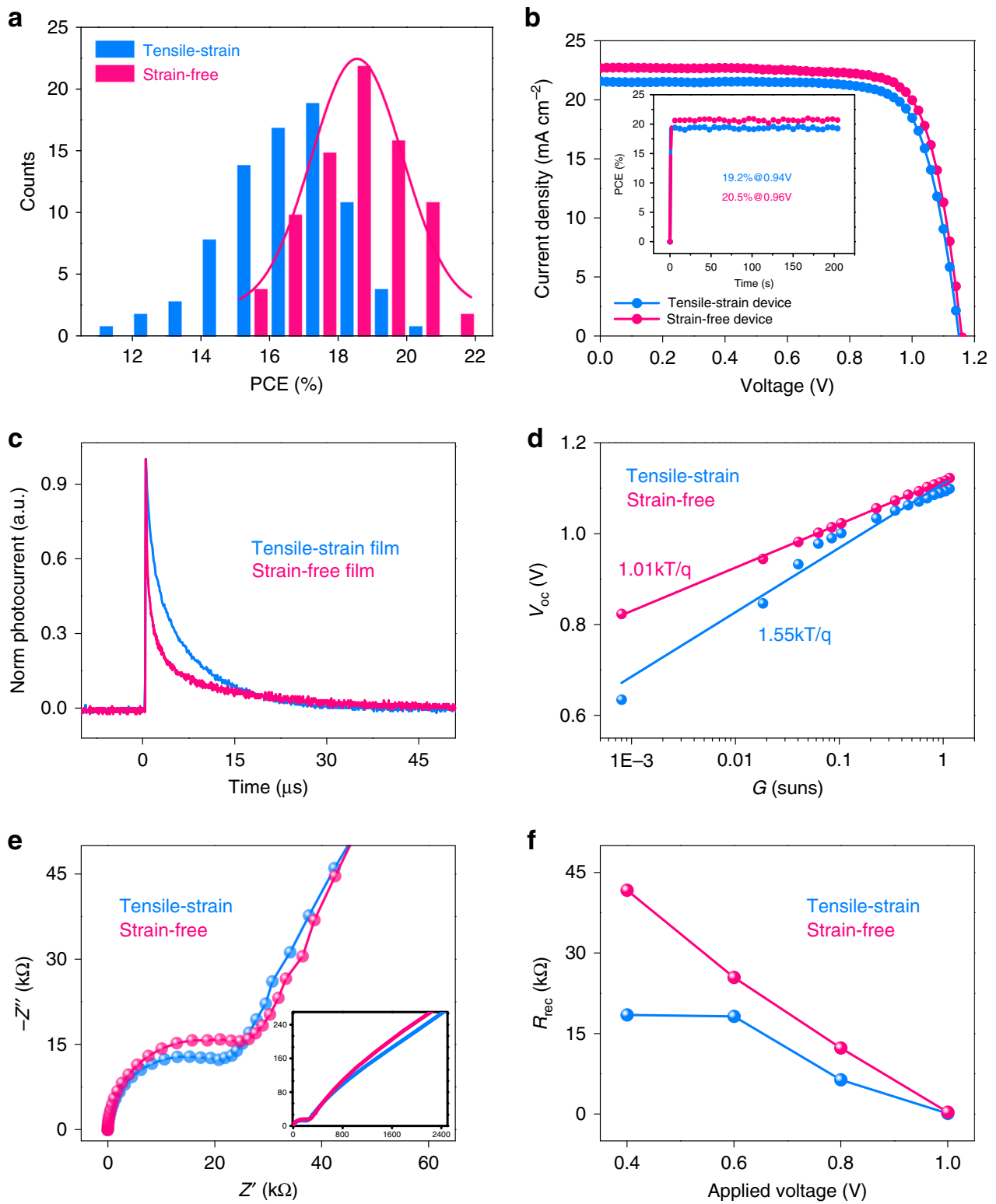

Fig. 3 Device performance and carrier dynamic behavior analysis. a Histograms of the PCEs for the devices with different strain conditions. $\mathbf{b} J-V$ curves of the tensile strain device and strain-free device. The inset is the stabilized current density measured at a bias voltage $(0.94,0.96 \mathrm{~V}$, respectively). c TPC decay curves for PSCs with tensile strain and strain-free conditions. $\mathbf{d}$ The light-intensity dependence of $V_{O C}$ measurement related to tensile strain and strain-free device. e EIS curves for PSCs with different strain conditions and the inset is frequency response signal according to frequency parameter from 1 $\mathrm{MHz}$ to $100 \mathrm{~Hz}$. $\mathbf{f}$ Variation of recombination resistance as a function of applied voltage. PCE power conversion efficiency, TPC transient photocurrent, ElS electrochemical impedance spectroscopy

zero, to tensile biaxial strain, the in-plane lattice gradually expands, which weakens the $\mathrm{Pb}-\mathrm{X}$ bonds and thus in principle pulls down both the anti-bonding VB and $\mathrm{CB}$ energy levels. However, the VB with strong anti-bonding hybridization is substantially decreased, while the $\mathrm{CB}$ is less affected. As the result, the bandgap shows increase from compressive, zero, to tensile strain. This explanation is indeed supported by our calculations as shown in Fig. 4a.

Based on the changed electronic structure in the strained perovskite, we attempt to illustrate how residual strains affect hole carrier dynamics in solar cells. Figure $4 \mathrm{~b}$ (left panel) shows the evolution of band-edge energies under tensile strains. The $\mathrm{CB}$ decreases slightly with increasing strain magnitude, whereas the VB exhibits pronounced downshift. It reveals that strains evolve vertically in the perovskite film, wherein the largest tensile strain is observed at the perovskite/HTM interface. Therefore, the VB bends downward monotonously over the entire perovskite absorber layer, as depicted in the Fig. $4 \mathrm{~b}$ (right panel). This VB downward bending has dual effects on the hole carrier dynamics. On one hand, it creates the "cliff-type" band alignment by repelling hole carrier energy level away from that of the HTM (Fig. 4b). This was reported to be unfavorable for hole extraction in $\mathrm{Cu}(\mathrm{In}, \mathrm{Ga}) \mathrm{Se}_{2}{ }^{53}$ and perovskite solar cells ${ }^{54-58}$. On the other hand, the hole mobility is possibly affected along the direction of VB downward bending, wherein the hole carrier diffusion suffers from extra hindering force field due to unfavored energy level gradient. As indicated by the space-charge-limited current (SCLC) measurement (Fig. 4d) of the fabricated capacitor-like 


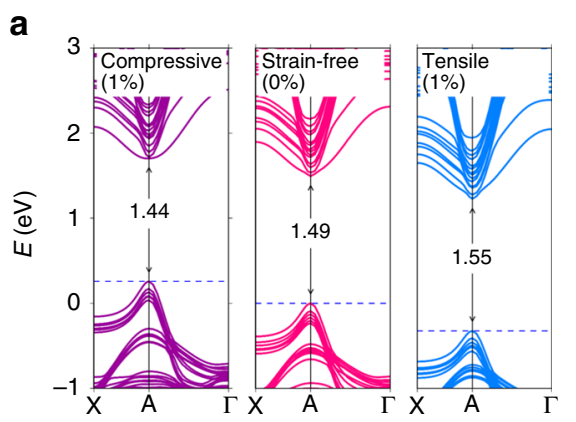

b

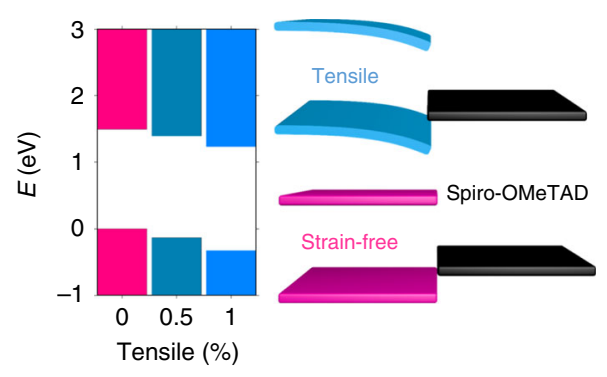

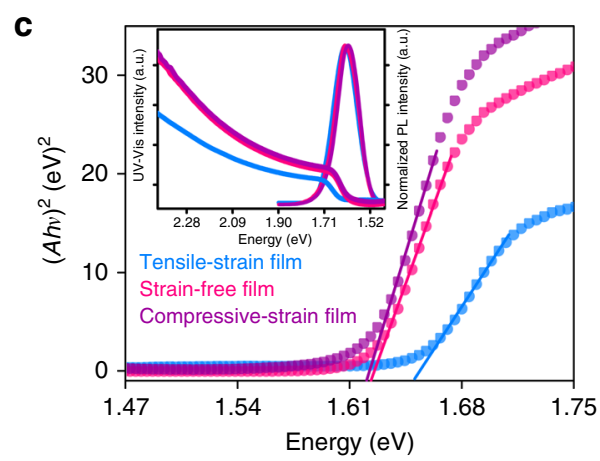

d

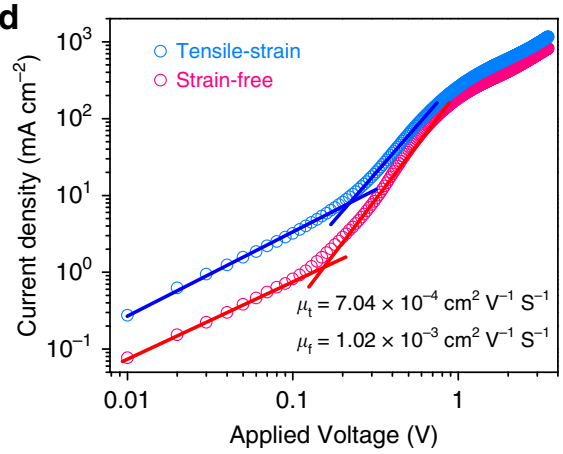

Fig. 4 Strain-induced electronic structure analysis. a Calculated band structures under biaxial tensile, zero, and compressive strains from first-principle density functional theory (DFT)-based approaches. The band structure alignment is made by using the vacuum energy level as reference. $\mathbf{b}$ The evolution of band-edge energies under gradually increasing tensile strains in perovskite films (left panel), and the schematic of the band alignment between tensile strain/strain-free film and hole transport layer in solar cell. c Ultraviolet (UV)-visible (Vis) absorption spectra and PL spectra under tensile strain, strainfree, and compressive strain conditions. d The J-V characteristics of the hole-only space-charge-limited current (SCLC) device with/without residual tensile strain

devices by sandwiching the perovskite films between ITO and $\mathrm{Au}$, the mobilities of the samples with and without tensile strain were calculated to be $7.04 \times 10^{-4}$ and $1.02 \times 10^{-3} \mathrm{~cm}^{2} \mathrm{~V}^{-1} \mathrm{~S}^{-1}$, respectively. Indeed, the experimentally observed carrier mobility is improved when tensile strain is released. In short, by eliminating the tensile strain gradient in the perovskite film, the VB is flattened to cancel the "cliff-type" band alignment of perovskite absorber/HTM and hole mobility is enhanced simultaneously. It thus favors the charge transport and extraction of photogenerated holes, which suppresses the carrier recombination and leads to the significant improvement in $\mathrm{FF}$ and $V_{\mathrm{OC}}$ in the corresponding device.

In addition to the above two main effects, the tensile strain induced downward shift of valence bands may also result in the deeper defect levels of the perovskite films with the assumption of defect energy levels being not sensitive to strain. This is supported by the experimental observation that the hydrostatic pressure render the shallower defect energy levels of hybrid halide perovskites ${ }^{59,60}$. As demonstrated above we also observed the prolonged carrier lifetime in the strain-free samples (Supplementary Fig. 14).

\section{Discussion}

In conclusion, we revealed gradient evolution of residual strain in the vertical direction of the mixed halide perovskite film by depth-dependent grazing incident X-ray diffraction characterization. The residual strain distribution may be stemmed from composition inhomogeneity and/or gradient thermal stress during film processing. A simple technique was developed to modulate the strain nature (e.g., tensile and compressive) and its gradient over the perovskite film in a controllable manner. This allows us to identify substantial impact of the residual strain gradient on hole carrier dynamics across the perovskite solar cell. First-principle calculations reveal that the strain gradient induces valence bands bending of perovskite absorber and thus affects the interfacial hole dynamics. By reducing the strain gradient of the perovskite film through strain engineering, we achieved substantial improvement in hole carrier transport and extraction across the interface of perovskite absorber/HTM. Consequently, the optimized perovskite solar cell reaches the certified power conversion efficiency of $20.7 \%$. By demonstrating the impact of residual strain on optoelectronic properties of halide perovskite film, this contribution sheds a light on further understanding the composition-structure-property relationship of halide perovskite system, which may be exploited to further advance the performance of halide perovskite based optoelectronic devices.

\section{Methods}

Materials. All the commercial materials were used as received without further purification, including ethanol (AR Beijing Chemical Works), Methylamine (33 wt.\% in absolute ethanol), Formamidine acetate (99\%, Aldrich), $\mathrm{HBr}$ (48 wt.\% in water, Sigma-Aldrich), HI (57 wt.\% in water, Sigma-Aldrich), $\mathrm{PbI}_{2}$ (99.999\%, SigmaAldrich), $\mathrm{PbBr}_{2}$ (99.999\%, Aldrich), CsI (99.90\%, Aladdin Industrial Corporation) $\mathrm{N}$, N-dimethylformamide (DMF, 99.99\%, Sigma-Aldrich), Dimethyl sulfoxide (DMSO, 99.9\%, Sigma-Aldrich), chlorobenzene (CB, 99.9\%, Sigma-Aldrich), Spiro-OMeTAD (Lumtec), bis(trifluoromethane)sulfonimide lithium salt (99.95\%, Aldrich), 4-tertbutylpyridine (99.9\%, Sigma-Aldrich), acetonitrile (99.9\%, Sigma-Aldrich), and ITO substrates. The $\mathrm{HC}\left(\mathrm{NH}_{2}\right)_{2} \mathrm{I}$ and $\mathrm{CH}_{3} \mathrm{NH}_{3} \mathrm{Br}$ were prepared according to procedure mentioned in previous work.

Perovskite precursor solutions. The precursor solutions were prepared according to the work delivered by Saliba.

FAMA perovskite solution: The mixed perovskite solution were prepared by mixing FAI (1.38 M), $\mathrm{PbI}_{2}(1.49 \mathrm{M}), \mathrm{MABr}(0.24 \mathrm{M}), \mathrm{PbBr}_{2}(0.26 \mathrm{M})$ in anhydrous DMF: DMSO 4:1 (v:v) according to previous work with a slight amount of 
excessive $\mathrm{PbI}_{2}$. To be convenient, we labeled the mixed perovskite solution with compositions mentioned above as FAMA ${ }^{57}$

CsI solutions: CsI solution was deposited by dissolving CsI in pure DMSO with the concentration of $1.5 \mathrm{M}$.

$(\text { FAMA })_{(100-x)} \mathrm{Cs}_{x}$ perovskite solution: (FAMA $)_{(100-x)} \mathrm{Cs}_{x}$ perovskite solution was obtained by adding appropriate amount of CsI into $300 \mu \mathrm{L}$ FAMA perovskite solution with different cesium concentrations (volume ratio, $x=100 \% \times V_{\mathrm{CsI}} /$ $\left.\left(V_{\text {CsI }}+300\right)\right)$ to achieve the desired cation composition $5 \%$.

\section{Optimal flipped annealing method for polycrystalline perovskite film. The} perovskite film washed by antisolvent was annealed about $20 \mathrm{~min}$ at $120^{\circ} \mathrm{C}$ through the normal method in order to most of the solvent can escape smoothly, then it was flipped over and annealed $25 \mathrm{~min}$ at $120^{\circ} \mathrm{C}$ so that the maximum tensile strain in the top surface can be gradually released at the highest temperature. In order to drop down the cooling rate at the same total annealing time, we first transferred the film to a hot stage at $80^{\circ} \mathrm{C}$ for $1 \mathrm{~min}$ and then transferred to a hot stage at $40^{\circ} \mathrm{C}$ for $1 \mathrm{~min}$. All annealing processes are finished in in a nitrogen glove box.

Sample preparation and devices fabrication. The ITO substrate was sequentially washed with distilled water, acetone, ethanol, and isopropanol. After $30 \mathrm{~min}$ of $\mathrm{UV}-\mathrm{O}_{3}$ treatments, the $\mathrm{SnO}_{2}$ electron transport layers (ETLs) were spin-coated on ITO substrates from the $\mathrm{SnO}_{2}$ colloidal solutions, and annealed on a hot plate at the displayed temperature of $150{ }^{\circ} \mathrm{C}$ for $30 \mathrm{~min}$ in ambient air. For the mixed Acation $\mathrm{FA}_{0.85} \mathrm{MA}_{0.15} \mathrm{~Pb}\left(\mathrm{I}_{0.85} \mathrm{Br}_{0.15}\right)_{3}$ and $\mathrm{FA}_{0.85} \mathrm{MA}_{0.15} \mathrm{Cs}_{0.05} \mathrm{~Pb}\left(\mathrm{I}_{0.85} \mathrm{Br}_{0.15}\right)_{3}$ metal halide perovskite layer, one-step method used toluene as antisolvent developed by Saliba was adopted. In detail, the perovskite solutions were spin-coated at $6000 \mathrm{rpm}$ for $30 \mathrm{~s}$. Two-hundred microliters of chlorobenzene was dropped on the spinning substrate during the spin-coating step at $10 \mathrm{~s}$ before the end of the procedure. Then, the as-fabricated film were baked at $120^{\circ} \mathrm{C}$ for $45 \mathrm{~min}$ in a nitrogen filled glove box. After the perovskite annealing, $30 \mu \mathrm{L}$ Spiro-OMeTAD solution doped with LiTFSI and tBP was deposited at $3000 \mathrm{rpm}$ for $30 \mathrm{~s}$. The hole transport material (HTM) solution was prepared by dissolving $60 \mathrm{mg}$ spiro-OMeTAD, $30 \mu \mathrm{L}$ 4-tert-butylpyridine and $35 \mu \mathrm{L} \mathrm{Li-TFSI/acetonitrile}\left(260 \mathrm{mg} \mathrm{mL}^{-1}\right)$ in $1 \mathrm{~mL}$ chlorobenzene. Finally, $100 \mathrm{~nm} \mathrm{Ag}$ was thermally evaporated as counter electrode under a pressure of $5 \times 10^{-5} \mathrm{~Pa}$ on top of the hole transport layer to form the back contact.

Fabrication of space-charge limited current devices. The structure of the device was ITO/PEDOT:PSS/perovskite/Spiro-OMeTAD /Au. The ITO glass substrate was treated by $\mathrm{UV}-\mathrm{O}_{3}$ for $30 \mathrm{~min}$, the PEDOT:PSS was filtered and then spincoated on ITO substrates. $\mathrm{FA}_{0.85} \mathrm{MA}_{0.15} \mathrm{PbI}_{2.55} \mathrm{Br}_{0.45}$ perovskite film was deposited on the PEDOT:PSS/ITO substrate by spin-coating. After the perovskite annealing, Spiro-OMeTAD layer was deposited and $150 \mathrm{~nm}$ Au was thermally evaporated as counter electrode.

Characterizations. The morphologies and sizes of nanocrystals were characterized by JEOL JEM-2100 transmission electron microscopy (TEM). Scanning electron microscope (SEM) images were measured using Hitachi S4800 fieldemission scanning electron microscopy. XRD patterns were recorded on a Rigaku smartlab X-ray Diffractometer. The cross-section of the device was prepared by focused ion beam (FIB) using a FEI Helios Dualbeam system. The sample was first covered by Pt protection layer deposited by electron beam and ion beam in dualbeam system, and was then milled to thin lamella following standard FIB sample preparation techniques. Due the beam-sensitivity of organic-inorganic hybrid halide perovskite structure, low beam voltage, and current was applied during final cleaning steps. The as-prepared thin lamella was studied by high angle annular dark field scanning transmission electron microscopy (HAADF-STEM) using a FEI Titan G2 microscope equipped by an aberration corrector for probe forming lens, operated at $300 \mathrm{kV}$. Depthdependent steady-state photoluminescence (PL) measurement was executed by confocal fluorescence microscope and time-resolved photoluminescence data was obtained by FLS980 (Edinburgh Instruments Ltd) with an excitation at 465 $\mathrm{nm}$. The time-of-flight secondary ion mass spectrometry (TOF-SIMS) measurements (Model TOF-SIMS V, ION-TOF GmbH) were performed with the pulsed primary ions from a Cs+ $(3 \mathrm{keV})$ liquid-metal ion gun and a $\mathrm{Bi}+$ pulsed primary ion beam for the analysis $(25 \mathrm{keV})$. Current-voltage characteristics were recorded by using a Keithley 2400 source-measure unit. The typical current-voltage characteristics of the devices were measured using a Keithley 2400 source meter by reverse scanning from 1.2 to $-0.2 \mathrm{~V}$ or forward scanning from -0.2 to $1.2 \mathrm{~V}$ at a scanning speed of $50 \mathrm{mV} \mathrm{s}^{-1}$. The photocurrent was measured under AM1.5G illumination at $100 \mathrm{~mW} \mathrm{~cm}^{-2}$ under a by $3 \mathrm{~A}$ steadystate solar simulator (SS-F5-3A). Light-intensity was calibrated with a National Institute of Metrology (China) calibrated KG5-filtered Si reference cell. The effective area of each cell was $0.102 \mathrm{~cm}^{2}$ defined by masks for all the photovoltaic devices discussed in this work. Space-charge limited current measurements were conducted on hole-only ITO/PEDOT:PSS/perovskite/Spiro-OMeTAD/Ag devices. A Keithly 2400 source meter was used to measure the relevant $J-V$ curves. The absorption spectra were measured by Hitachi UH4150 spectrophotometer.
External quantum efficiencies (EQE) were performed on a solar cell quantum efficiency measurement system (QE-R) supported by Enli Technology Co., Ltd. A calibrated silicon diode with a known spectral response was used as a reference. The transient photovoltage/photocurrent (TPV/TPC) decay measurements were obtained on Molex 180081-4320 simulating one sun working condition, the carriers were excited by a $532 \mathrm{~nm}$ pulse laser. The electrochemical impedance spectroscopy (EIS) was determined by the electrochemical workstation (Germany, Zahner Company), employing light emitting diodes driven by Export (Germany, Zahner Company).

First-principles calculations. Calculations were performed within the framework of density functional theory (DFT) by using plane-wave pseudopotential methods as implemented in the Vienna Ab initio Simulation Package ${ }^{61,62}$. The electron-ion interactions were described by the projected augmented wave pseudopotentials ${ }^{63}$ with the $1 s(\mathrm{H}), 2 s$ and $2 p(\mathrm{C}), 2 s$ and $2 p(\mathrm{~N}), 5 s$ and $5 p(\mathrm{I})$ and $5 d, 6 s$ and $6 p(\mathrm{~Pb})$ electrons treated explicitly as valence electrons. We used the generalized gradient approximation formulated by Perdew, Burke, and Ernzerhof ${ }^{64}$ as the exchange correlation functional. Kinetic Energy cutoff for the plane-wave basis set were set to $400 \mathrm{eV}$. The k-point meshes with grid spacing of $2 \pi \times 0.03 \AA^{-1}$ were used for electronic Brillouin zone integration. We modeled the perovskite film by using the (001)-oriented six-layers Ruddlesden-Popper phase of $\mathrm{FAPbI}_{3}$ embedded in a vacuum region of $30 \AA$. The biaxial strains of $1 \%, 0.5 \%$ (compressive) and $-0.5 \%,-1 \%$ (tensile) were applied to the film, respectively. The structures under the biaxial strain conditions were optimized by fixing the in-plane structure parameters of $\mathrm{Pb}-\mathrm{I}$ perovskite framework through total energy minimization with the residual forces on the atoms converged to below $0.02 \mathrm{eV}^{-1}$. To properly take into account the long-range van der Waal interactions that play a nonignorable role in the hybrid perovskites involving organic molecules, the vdW-optB86b functional ${ }^{65}$ was adopted. The alignment of the band structures upon different strains is made by using the vacuum energy as reference.

\section{Data availability}

All the relevant data are available from the corresponding authors upon reasonable request.

Received: 16 August 2018 Accepted: 6 January 2019 Published online: 18 February 2019

\section{References}

1. Jeon, N. J. et al. Compositional engineering of perovskite materials for highperformance solar cells. Nature 517, 476-480 (2016).

2. Jeon, N. J. et al. Solvent engineering for high-performance inorganicorganic hybrid perovskite solar cells. Nat. Mater. 13, 897-903 (2014).

3. Saliba, M. et al. Cesium-containing triple cation perovskite solar cells: improved stability, reproducibility and high efficiency. Energy Environ. Sci. 9, 1989 (2016)

4. Snaith, H. J. et al. Present status and future prospects of perovskite photovoltaics. Nat. Mater. 17, 372-376 (2018).

5. Xiao, J. W. et al. The emergence of the mixed perovskites and their applications as solar cells. Adv. Energy Mater. 7, 1700491 (2017).

6. Malinkiewicz, O. et al. Perovskite solar cells employing organic chargetransport layers. Nat. Photon. 8, 128 (2013).

7. Yang, W. S. et al. Iodide management in formamidinium-lead-halide-based perovskite layers for efficient solar cells. Science 356, 1376-1379 (2017).

8. $\mathrm{Li}, \mathrm{X}$. et al. A vacuum flash-assisted solution process for high-efficiency largearea perovskite solar cells. Science 353, 58-62 (2016).

9. $\mathrm{Wu}, \mathrm{W}$. Q. et al. Molecular doping enabled scalable blading of efficient hole-transport-layer-free perovskite solar cells. Nat. Commun. 9, 1625 (2018)

10. He, M. et al. Meniscus-assisted solution printing of large-grained perovskite films for high-efficiency solar cells. Nat. Commun. 8, 16045 (2017).

11. Li, Z. et al. Scalable fabrication of perovskite solar cells. Nat. Rev. Mater. 3, 18017 (2018).

12. Eperon, G. E. et al. Formamidinium lead trihalide: a broadly tunable perovskite for efficient planar heterojunction solar cells. Energy Environ. Sci. 7, 982-988 (2014).

13. Jodlowski, A. D. et al. Large guanidinium cation mixed with methylammonium in lead iodide perovskites for $19 \%$ efficient solar cells. Nat. Energy 2, 972 (2017).

14. Huang, $\mathrm{Y}$. et al. The intrinsic properties of $\mathrm{FA}_{(1-\mathrm{x})} \mathrm{MA}_{\mathrm{x}} \mathrm{PbI} 3$ perovskite single crystals. J. Mater. Chem. A 5, 8537-8544 (2017).

15. Saparov, B. et al. Organic-inorganic perovskites: Structural versatility for functional materials design. Chem. Rev. 116, 4558 (2016). 
16. Gratia, P. et al. Intrinsic halide segregation at nanometer scale determines the high efficiency of mixed cation/mixed halide perovskite solar cells. J. Am. Chem. Soc. 138, 15821-15824 (2016).

17. Philippe, B. et al. Chemical distribution of multiple cation $\left(\mathrm{Rb}^{+}, \mathrm{Cs}^{+}, \mathrm{MA}^{+}\right.$, and $\mathrm{FA}^{+}$) perovskite materials by photoelectron spectroscopy. Chem. Mater. 29, 3589-3596 (2017).

18. Abdi-Jalebi, M. et al. Maximizing and stabilizing luminescence from halide perovskites with potassium passivation. Nature 555, 497 (2018).

19. Brennan, M. C. et al. Light-induced anion phase segregation in mixed halide perovskites. ACS Energy Lett. 3, 204-213 (2018).

20. Bush, K. A. et al. Controlling thin-film stress and wrinkling during perovskite film formation. ACS Energy Lett. 3, 1225-1232 (2018).

21. Zheng, X. et al. Improved phase stability of formamidinium lead triiodide perovskite by strain relaxation. ACS Energy Lett. 1, 1014-1020 (2016).

22. Zhao, J. et al. Strained hybrid perovskite thin films and their impact on the intrinsic stability of perovskite solar cells. Sci. Adv. 3, eaao5616 (2017).

23. Prasanna, R. et al. Band gap tuning via lattice contraction and octahedral tilting in perovskite materials for photovoltaics. J. Am. Chem. Soc. 139, 11117-11124 (2017).

24. Murali, B. et al. Temperature-induced lattice relaxation of perovskite crystal enhances optoelectronic properties and solar cell performance. J. Phys. Chem. Lett. 8, 137-143 (2017).

25. MD, P. et al. Crystallization kinetics and morphology control of formamidinium-cesium mixed-cation lead mixed-halide perovskite via tunability of the colloidal precursor solution. Adv. Mater. 29, 1607039 (2017).

26. Feng, J. et al. Strain-engineered artificial atom as a broad-spectrum solar energy funnel. Nat. Photon. 6, 866-872 (2012).

27. Kurdi, M. E. et al. Control of direct band gap emission of bulk germanium by mechanical tensile strain. Appl. Phys. Lett. 96, 1738 (2010).

28. Ahn, G. H. et al. Strain-engineered growth of two-dimensional materials. Nat. Commun. 8, 608 (2017).

29. Hubbard, S. M. et al. Effect of strain compensation on quantum dot enhanced GaAs solar cells. Appl. Phys. Lett. 92, 5014-5166 (2008).

30. Oshima, R. et al. Strain-compensated InAs/GaNAs quantum dots for use in high-efficiency solar cells. Appl. Phys. Lett. 93, 083111 (2008).

31. Leblebici, S. Y. et al. Facet-dependent photovoltaic efficiency variations in single grains of hybrid halide perovskite. Nat. Energy 1, 16093 (2016)

32. Vailionis, A. et al. Misfit strain accommodation in epitaxial A B O 3 perovskites: Lattice rotations and lattice modulations. Phys. Rev. B 83, 064101 (2011).

33. Benediktovitch, A. et al. Stress gradient analysis by noncomplanar X-ray diffraction and corresponding refraction correction. Adv. Mater. Res $\mathbf{9 9 6}$ 162-168 (2014).

34. Stefenelli, M. et al. X-ray analysis of residual stress gradients in TiN coatings by a Laplace space approach and cross-sectional nanodiffraction: a critical comparison. J. Appl. Crystallogr. 46, 1378-1385 (2013).

35. Chen, Z. et al. Residual stress gradient analysis with GIXRD on $\mathrm{ZrO} 2$ thin films deposited by MOCVD. Surf. Coat. Technol. 206, 405-410 (2011).

36. Zhumekenov, A. A. et al. Formamidinium lead halide perovskite crystals with unprecedented long carrier dynamics and diffusion length. Acs Energy Lett. 1, 32-37 (2016)

37. Ahn, N. et al. Highly reproducible perovskite solar cells with average efficiency of $18.3 \%$ and best efficiency of $19.7 \%$ fabricated via lewis base adduct of lead (II) iodide. J. Am. Chem. Soc. 137, 8696 (2015).

38. Zhifang, S. et al. Symmetrization of the crystal lattice of $\mathrm{MAPbI}_{3}$ boosts the performance and stability of metal-perovskite photodiodes. Adv. Mater. 29, 1701656 (2017).

39. Nah, S. et al. Spatially segregated free-carrier and exciton populations in individual lead halide perovskite grains. Nat. Photon. 11, 285-288 (2017)

40. Wright, A. D. et al. Electron-phonon coupling in hybrid lead halide perovskites. Nat. Commun. 7, 11755 (2016).

41. Leijtens, T. et al. Towards enabling stable lead halide perovskite solar cells; interplay between structural, environmental, and thermal stability. J. Mater. Chem. A 5, 11483-11500 (2017).

42. Yin, W.-J. et al. Unusual defect physics in $\mathrm{CH} 3 \mathrm{NH} 3 \mathrm{PbI} 3$ perovskite solar cell absorber. Appl. Phys. Lett. 104, 063903 (2014).

43. Xiao, Z. et al. Progress in theoretical study of metal halide perovskite solar cell materials. Adv. Energy Mater. 7, 1701136 (2017).

44. Chen, H. et al. Molecular engineered hole-extraction materials to enable dopant-free, efficient p-i-n perovskite solar cells. Adv. Energy Mater. 7, 1700012 (2017).

45. Rajagopal, A. et al. Highly efficient perovskite-perovskite tandem solar cells reaching $80 \%$ of the theoretical limit in photovoltage. Adv. Mater. 29, 1702140 (2017).

46. Sherkar, T. et al. Recombination in perovskite solar cells: Significance of grain boundaries,interface traps, and defect ions. Acs Energy Lett. 2, 1214-1222 (2017).
47. Pankaj, Y. et al. The role of rubidium in multiple-cation-based high-efficiency perovskite solar cells. Adv. Mater. 29, 1701077 (2017)

48. $\mathrm{Li}, \mathrm{H}$. et al. Carbon quantum dots/TiOx electron transport layer boosts efficiency of planar heterojunction perovskite solar cells to $19 \%$. Nano Lett. 17 2328 (2017).

49. Zhou, $\mathrm{H}$. et al. Photovoltaics. Interface engineering of highly efficient perovskite solar cells. Science 345, 542-546 (2014)

50. Yang, D. et al. Functionality-directed screening of Pb-free hybrid organicinorganic perovskites with desired intrinsic photovoltaic functionalities. Chem. Mater. 29, 524-538 (2017).

51. Yin, W. J. et al. Halide perovskite materials for solar cells: a theoretical review. J. Mater. Chem. A 3, 8926-8942 (2015).

52. Brandt, R. E. et al. Identifying defect-tolerant semiconductors with high minority-carrier lifetimes: beyond hybrid lead halide perovskites. MRS Commun. 5, 265-275 (2015).

53. Giorgi, G. et al. Small photocarrier effective masses featuring ambipolar transport in methylammonium lead iodide perovskite: A density functional analysis. J. Phys. Chem. Lett. 4, 4213-4216 (2013).

54. HS, N. et al. Investigating the role of 4-tert butylpyridine in perovskite solar cells. Adv. Energy Mater. 7, 1601079 (2017).

55. Kobayashi, T. et al. $\mathrm{Cu}(\mathrm{In}, \mathrm{Ga}) \mathrm{Se}_{2}$ thin film solar cells with a combined ALD $\mathrm{Zn}(\mathrm{O}, \mathrm{S})$ buffer and MOCVD-ZnO:B window layers. Sol. Energy Mater. Sol. Cells 119, 129-133 (2013)

56. Chen, Q. et al. Controllable self-induced passivation of hybrid lead iodide perovskites toward high performance solar cells. Nano Lett. 14, 4158 (2014).

57. Jeng, J. Y. et al. $\mathrm{CH} 3 \mathrm{NH} 3 \mathrm{PbI} 3$ perovskite/fullerene planar-heterojunction hybrid solar cells. Adv. Mater. 25, 3727-3732 (2013).

58. Hawash, Z. et al. Interfacial modification of perovskite solar cells using an ultrathin MAI layer leads to enhanced energy level alignment, efficiencies, and reproducibility. J. Phys. Chem. Lett. 8, 3947-3953 (2017).

59. Kong, L. et al. Simultaneous band-gap narrowing and carrier-lifetime prolongation of organic-inorganic trihalide perovskites. Proc. Natl Acad. Sci. U.S.A 113, 8910-8915 (2016)

60. Gang, L. et al. Pressure-induced bandgap optimization in lead-based perovskites with prolonged carrier lifetime and ambient retainability. Adv. Funct. Mater. 27, 1604208 (2017).

61. Kresse, G. et al. Efficiency of ab-initio total energy calculations for metals and semiconductors using a plane-wave basis set. Comp. Mater. Sci. 6, 15-50 (1996).

62. Kresse, G. et al. Efficient iterative schemes for ab initio total-energy calculations using a plane-wave basis set. Phys. Rev. B 54, 11169-11186 (1996).

63. Blöchl, P. E. Projector augmented-wave method. Phys. Rev. B 50, 17953-17979 (1994).

64. Perdew, J. P. et al. Generalized gradient approximation made simple. Phys. Rev. Lett. 77, 3865-3868 (1996).

65. Klimeš, J. et al. Van der Waals density functionals applied to solids. Phys. Rev. B 83, 195131 (2011).

\section{Acknowledgements}

We acknowledge funding support from National Key Research and Development Program of China Grant No. 2016YFB0700700, National Natural Science Foundation of China (51673025 and 51672008), and the Young Talent Thousand Program. L.Z. acknowledges the support of the NSFC (Grant 61722403 and 11674121), National Key Research and Development Program of China (Grant 2016YFB0201204), and Program for JLU Science and Technology Innovative Research Team. P.C. acknowledges the support of the Project of State Key Laboratory of Explosion Science and Technology, Beijing Institute of Technology under Grant ZDKT18-01. Calculations were performed in part at High Performance Computing Center of Jilin University. S.Y. and B.H. appreciate Shenzhen Peacock Plan (KQTD2016053015544057) and Nanshan Pilot Plan (LHTD20170001). We appreciate the insightful technical discussion and experimental support with Mr. Gang Tang and Mr. Yizhou Zhao. We would like to thank Enli Technology for providing the depth-dependent PL measurement and EQE measurement.

\section{Author contributions}

Q.C. and C.Z. conceived the idea and designed the experiments. Both C.Z. and X.N. were involved in all the experimental parts. N.L, H.C.Z. and Y.C carried out the PL measurement, P.L. finished EIS measurements, G.Y. and Y.L. finished TEM measurements. X.K. and M.S. provided the insightful technical assistance for TEM analysis. Y.F., X.H. and G.N performed the theoretical calculations, L.Z. and Y.F. analyzed the results. S.Y., Y.B. and C.H. performed the TOF-SIM measurement. P.C. provided theoretical support for firstprinciples calculations. All the authors were involved in the discussion for data analysis and commented on the manuscript, and Y.L., L.Z., H.Z., Q.C. and C.Z. co-wrote the paper 


\section{Additional information}

Supplementary Information accompanies this paper at https://doi.org/10.1038/s41467019-08507-4

Competing interests: The authors declare no competing interests.

Reprints and permission information is available online at http://npg.nature.com/ reprintsandpermissions/

Journal peer review information: Nature Communications thanks Anna Osherov and the other anonymous reviewer(s) for their contribution to the peer review of this work.

Publisher's note: Springer Nature remains neutral with regard to jurisdictional claims in published maps and institutional affiliations. (c) (i) Open Access This article is licensed under a Creative Commons Attribution 4.0 International License, which permits use, sharing, adaptation, distribution and reproduction in any medium or format, as long as you give appropriate credit to the original author(s) and the source, provide a link to the Creative Commons license, and indicate if changes were made. The images or other third party material in this article are included in the article's Creative Commons license, unless indicated otherwise in a credit line to the material. If material is not included in the article's Creative Commons license and your intended use is not permitted by statutory regulation or exceeds the permitted use, you will need to obtain permission directly from the copyright holder. To view a copy of this license, visit http://creativecommons.org/ licenses/by/4.0/.

(C) The Author(s) 2019 